\title{
Key role of molecular kinetic energy in early stages of pentacene island growth
}

\author{
Yu Wu • Tullio Toccoli $\cdot$ Jian Zhang • Norbert Koch • \\ Erica Iacob • Alessia Pallaoro • Salvatore Iannotta • \\ Petra Rudolf
}

Received: 31 July 2008 / Accepted: 24 November 2008 / Published online: 8 January 2009

(C) The Author(s) 2009. This article is published with open access at Springerlink.com

\begin{abstract}
Organic molecular beam deposition is studied systematically at thermal and hyperthermal regimes aiming at investigating the role of molecular kinetic energy on the growth mechanism of pentacene submonolayers on $\mathrm{SiO}_{x} / \mathrm{Si}$. We show that the kinetic energy of the impinging molecule $\left(E_{k}\right)$ plays a crucial role in determining island structure and shape, distribution of island sizes, the crystalline quality of the first monolayer, and even the growth mode of subsequent layers. With increasing $E_{k}$, the island structure changes from fractal to nonfractal, the shape becomes more anisotropic and the island size more uniform, pointing to correlated island growth. Moreover, while 3D island growth is observed for thermal organic molecular beam de-
\end{abstract}

\footnotetext{
Y. Wu $\cdot$ P. Rudolf $(\bowtie)$

Zernike Institute for Advanced Materials,

University of Groningen, Nijenborgh 4, 9749 AG

Groningen, The Netherlands

e-mail: p.rudolf@rug.nl

Fax: 31-50-3634879

T. Toccoli $\cdot$ A. Pallaoro $\cdot$ S. Iannotta

IFN-CNR, CEFSA-FBK Institute of Photonics

and Nanotechnology, Trento, Via alla Cascata 56C,

38050 Povo, Trento, Italy

J. Zhang $\cdot$ N. Koch

Institut für Physik, Humboldt-Universität zu Berlin,

Newtonstrasse 15, 12489 Berlin, Germany

E. Iacob

FBK-Materials and Microsystems, Via Sommarive, 18, 38050 Povo, Trento, Italy

S. Iannotta

IMEM-CNR, Institute of Materials for Electronics and Magnetism, Parco Area delle Scienze 37/A, 43100 Parma, Italy
}

position, supersonic molecular beam deposition gives rise to layer-by-layer growth, at least for the first two layers. When $E_{k} \geq 5.0 \mathrm{eV}$, the first monolayer is composed of large single crystalline domains which can extend over up to $10 \mu \mathrm{m}$, inferred from comparing atomic force micrographs of height and net transverse shear force. In these growth conditions both the high surface diffusivity and energy redistribution play a major role. We propose a mechanism where the energy dissipation occurring during the molecule-surface collision leads to the reorientation of whole islands during island coalescence, resulting in the elimination of grain boundaries.

PACS 72.80.Le $\cdot 81.15-\mathrm{z} \cdot 79.20 \mathrm{Rf} \cdot 68.37 . \mathrm{Ps} \cdot 68.55 . \mathrm{J} \cdot$ 34.25.+a

\section{Introduction}

In comparison to other organic semiconductor materials, pentacene, exhibiting a high hole mobility of $5.5 \mathrm{~cm}^{2} / \mathrm{Vs}$ [1] in thin-film-based field-effect transistors (OTFTs), is widely regarded as one of the most promising candidates for plastic electronic application $[2,3]$. The charge carrier mobility is a crucial factor for the performance of electronic devices and previous studies have demonstrated that it is highly sensitive to the crystallinity of pentacene thin films, i.e., to grain size $[4,5]$, defect density $[6,7]$, and crystal polymorph $[8,9]$. Most studies have focused on the interface between pentacene and the $\mathrm{SiO}_{2}$ surface because, on one hand, the first layer acts as a template for subsequent layer growth and therefore determines the quality of successive pentacene layers and, on the other hand, the layers closest to the interface form the charge transport channel in OFETs [10].

Organic molecular beam deposition (OMBD), where pentacene is sublimed from a Knudsen cell, is so far the most 
commonly used method for growing thin films; however, recently supersonic molecular beam deposition (SuMBD) has proven to be a valuable alternative for this purpose $[11,12]$. In the past few years, several studies based on OMBD grown pentacene layers have demonstrated that the pentacene grain size can be modulated via changing the deposition rate, the substrate temperature or modifying the substrate surface with organic self assembled layers [13-17]. However, for SuMBD grown pentacene thin films, it is more straightforward to change the grain size by tuning the pentacene kinetic energy $\left(E_{k}\right)$ within the molecular beam. In recent studies on pentacene nucleation and growth, we showed how the pentacene critical nucleus size changes with the kinetic energy of the impinging molecules during the early phase of pentacene growth on $\mathrm{SiO}_{2} / \mathrm{Si}$ [18]. Here we extend this investigation to better understand the correlation between $E_{k}$ and island morphology. Three different molecular beams were chosen producing pentacene at kinetic energies of 3.3, 5.0, and $6.4 \mathrm{eV}$. OMBD films of similar coverage were grown for comparison. All samples were prepared with similar growth rate on hydrophilic $\mathrm{SiO}_{2} / \mathrm{Si}$ at room temperature. Ex situ tapping mode atomic force microscopy (TM-AFM) was used for investigating the sample morphology, and transverse shear microscopy (TSM) was employed to study the crystallinity of the first monolayer. We find that the correlated island growth becomes more pronounced with increasing $E_{k}$, i.e., the island structure changes from fractal to nonfractal. Although all investigated pentacene monolayers were polycrystalline, the single crystal grain size strongly depends on the kinetic energy of the impinging molecules and reaches $\sim 10 \mu \mathrm{m}$ when the samples are grown at $E_{k}=6.4 \mathrm{eV}$.

\section{Experimental methods}

\subsection{Substrates and substrate preparation}

$\mathrm{SiO}_{x}$ layers of $\sim 3-4 \mathrm{~nm}$ thickness, as measured by ellipsometry (Nanofilm Technology GmbH, Germany), were prepared by wet chemical oxidation of $10 \times 10 \mathrm{~mm}^{2}$ samples cut from a silicon wafer ( $\mathrm{Si}$ (100), p-type with boron dopant, resistivity 1 to $10 \Omega \mathrm{cm}^{-1}$, purchased from Silicon Quest International, USA). The as-cut substrates were cleaned by sonication in deionized water (Milli-Q, 18.0 M $\Omega$ ) and acetone for $15 \mathrm{~min}$, and then kept in boiling acetone for $15 \mathrm{~min}$. The chemical oxidation process included the following steps: Si wafer cuts were immersed for $30 \mathrm{~min}$ in $\mathrm{H}_{2} \mathrm{SO}_{4}: \mathrm{H}_{2} \mathrm{O}_{2}=1: 3$ solution, heated to $65^{\circ} \mathrm{C}$. After repeated rinsing with copious amounts of deionized water, the samples were transferred to $\mathrm{HCl}+\mathrm{H}_{2} \mathrm{O}_{2}(1: 1)$ piranha solution, heated to $25^{\circ} \mathrm{C}$ for $15 \mathrm{~min}$. Finally, samples were rinsed with deionized water and dried under nitrogen flow. The typical root-mean-square surface roughness was determined to be $\sim 0.5-1 \mathrm{~nm}$ by atomic force microscopy in tapping mode (TM-AFM), by which we also noticed the presence of a few bright spots (area density of $\sim 2.5 \times 10^{7} / \mathrm{cm}^{2}$ ), presumably due to contamination. Surface contact angles were measured in air using the sessile drop method [19] in a custom built microscope-goniometer system. A $1.25 \mu \mathrm{l}$ drop of deionized water was deposited onto the freshly prepared $\mathrm{SiO}_{x} / \mathrm{Si}$ substrate surface and the contact angle measured after 60 seconds. All substrates gave contact angles of $35 \pm 2^{\circ}$.

\subsection{Pentacene and pentacene film growth}

Pentacene (Sigma Aldrich, Corp., USA) was further purified by vacuum sublimation under a temperature gradient [20]. The purification was performed at $430 \mathrm{~K}$ for $70 \mathrm{~h}$ in the dark to protect against the UV degradation. The purified material was stored in the dark and low vacuum before use.

One set of pentacene samples was prepared by organic molecular beam deposition (OMBD) as described in [21]. The deposition rate was $0.4 \AA / \mathrm{min}$, as measured by a quartzcrystal microbalance. The substrate was kept at room temperature during deposition.

Three sets of pentacene samples were prepared by SuMBD as described in [22], with pentacene impinging on a surface area of $\approx 10 \mathrm{~mm}$ diameter, for different exposure times at room temperature. The molecular beam was carefully characterized on-line in terms of chemical purity, flux, and energy distribution by combining time of flight mass spectrometry (ToF-MS) and multiphoton ionization spectroscopy. We chose an operating regime where no clustering effects [23] and no detectable contaminants were present in the supersonic beam. The typical flux was about $6 \times 10^{11}$ pentacene molecules $/\left(\mathrm{s} \cdot \mathrm{cm}^{2}\right)$, estimated by cross correlating the ToF-MS spectra at different kinetic energies of the beam with those of the Knudsen source used as a standard. For the three deposition conditions chosen, the average molecular $E_{k}$ was $6.4,5.0$, and $3.3 \mathrm{eV}$, as deduced from the molecules' flight time. The growth rate was nearly the same for all three beams, amounting to about $1 \mathrm{ML}$ in $\mathbf{4 5 - 5 0 ~ m i n ~ a s ~ e s t i m a t e d ~}$ from ex situ TM-AFM measurements. This corresponds to approximately $0.3 \AA / \mathrm{min}$, a rate comparable to the one for OMBD growth.

\subsection{AFM characterization}

Samples were imaged by ex situ TM-AFM (Multimode Scanning Probe Microscope, Veeco Metrology Group, USA) with a silicon tip with nominal radius less than $10 \mathrm{~nm}$. We selected five scanning regions around the center of the deposition area and fixed the scanning size to $10 \times 10 \mu \mathrm{m}^{2}$. The detailed analysis of AFM data was carried out with the WSxM software (version 8.7, Nanotec Electronica S.L., 
Spain, see [24]). The statistical analysis of the AFM data was performed with the help of specific software (Origin 7.0, OriginLabs Inc., USA).

We also employed AFM to perform transverse shear microscopy (TSM) [25-27], where a silicon nitride tip (NP-20 Veeco Metrology and Instrument, USA) with a spring constant of $0.58 \mathrm{~N} \mathrm{~m}^{-1}$ is scanned in contact parallel to the cantilever's long axis. By mapping the variation in transverse shear force, we could qualitatively determine the crystallinity of a single molecular island or of the whole monolayer. $10 \times 10 \mu \mathrm{m}^{2}$ TSM images were collected in parallel with the TM-AFM height images for samples grown by OMBD and SuMBD presenting a nearly completed first monolayer.

\section{Results and discussion}

Figure 1 presents TM-AFM micrographs illustrating the morphology of pentacene layers corresponding to $\sim 0.3 \mathrm{ML}$ coverage and resulting from different growth conditions. All SuMBD samples (Fig. 1(b-d)) consist typically of lobular islands with smooth edges, while the OMBD sample (Fig. 1(a)) is characterized by islands with rougher edges and by the presence of smaller dendritic islands, as minority species. If one ignores the minority species, the samples grown by OMBD (Fig. 1(a)) and by SuMBD with $E_{k}=3.3 \mathrm{eV}$ (Fig. 1(b)) are rather similar, as they both show a larger average island size and lower nucleation density than the samples grown by SuMBD at $E_{k}=5.0 \mathrm{eV}$ and $E_{k}=6.4 \mathrm{eV}$, shown in Figs. 1(c) and 1(d), respectively.

The nucleation density $(N)$ as a function of the kinetic energy of the impinging molecules, plotted in Fig. 2(a), quantifies this observation. To derive $N$, the molecular island number was averaged from five AFM images collected in different regions on each sample. For the sample grown with $E_{k}=6.4 \mathrm{eV}, N$ is $\sim 57 \%$ higher than for the OMBD sample and $\sim 45 \%$ higher than for the SuMBD sample grown at $E_{k}=3.3 \mathrm{eV}$. It is only $\sim 5 \%$ higher than for the SuMBD sample grown at $E_{k}=5.0 \mathrm{eV}$. We suggest that this difference is due to a different molecular surface diffusivity due to the different growth regimes. In fact, the experimental evidence indicates that a pentacene monomer with high $E_{k}(\geq 5.0 \mathrm{eV})$ may sample many adsorption sites before being adsorbed or desorbed with roughly equal probabilities to either integrate into the preformed islands or form a new nucleus on the empty surface in between islands. For samples grown with pentacene at low $E_{k}$, such as in OMBD and SuMBD with $E_{k}=3.3 \mathrm{eV}$, the probability for an incoming monomer to irreversibly integrate into the preformed molecular islands becomes dominant with respect to forming a new nucleus in an empty substrate region [21]. Additionally, in our previous study of SuMBD

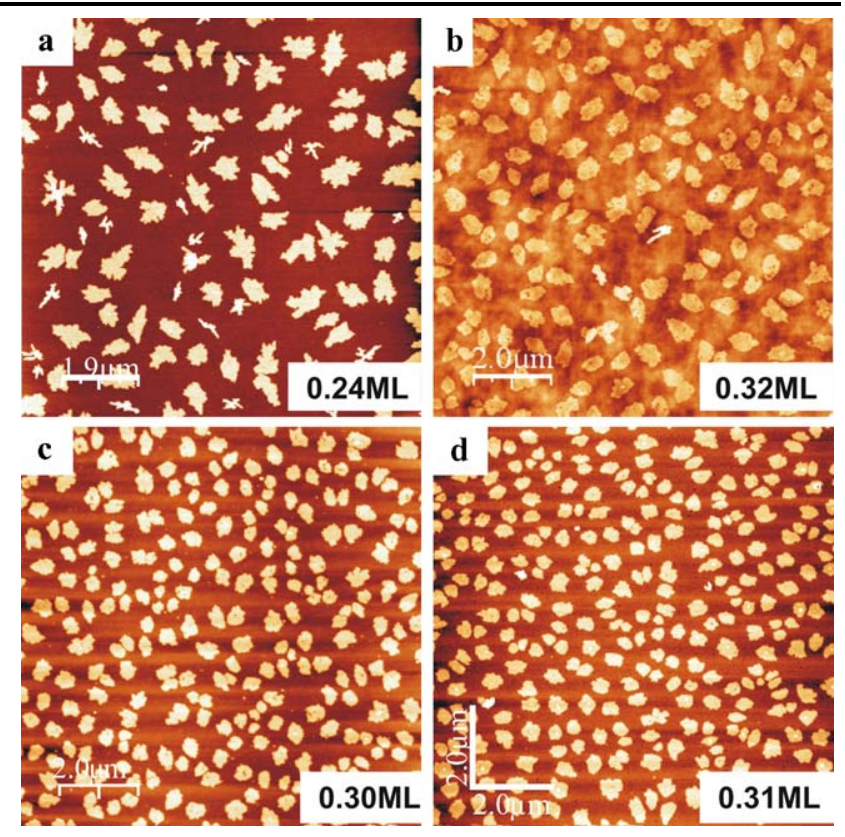

Fig. 1 Tapping mode AFM $\left(10 \times 10 \mu \mathrm{m}^{2}\right)$ micrographs of pentacene sub-monolayers grown by OMBD (a) and by SuMBD at $E_{k}=3.3 \mathrm{eV}(\mathbf{b}), E_{k}=5.0 \mathrm{eV}(\mathbf{c})$ and $E_{k}=6.4 \mathrm{eV}(\mathbf{d})$

grown pentacene on silicon oxide [18], we found that three pentacene molecules are needed to form a stable island in the growth regime where $E_{k}>5-6 \mathrm{eV}$, whereas four molecules are needed for $E_{k}<5-6 \mathrm{eV}$. Obviously, the probability that three free molecules with high surface diffusivity aggregate is higher than that for four free molecules with lower diffusivity. This is the basic mechanism that explains the high nucleation density with a dispersive nuclei distribution observed in the growth regime for $E_{k} \geq 5.0 \mathrm{eV}$ at low coverages.

We calculated the average island size for each sample from the sum of five island area histograms obtained from four AFM images collected in different regions of each sample. The sum histograms are presented in Fig. 2(b), and the average island size is derived by fitting the data with a lognormal function (also shown). We found average island sizes of $0.129 \mu \mathrm{m}^{2} \pm 0.002,0.116 \mu \mathrm{m}^{2} \pm 0.001$, and $0.108 \mu \mathrm{m}^{2} \pm 0.001$ for SuMBD samples with $E_{k}=3.3,5.0$, and $6.4 \mathrm{eV}$. An average island size of $0.216 \mu \mathrm{m}^{2} \pm 0.066$ was determined for the OMBD sample.

From Fig. 2 one infers that the island size distribution becomes narrower and peaked at smaller values as $E_{k}$ increases. This trend towards a more uniform island size is characteristic of a changeover towards correlated island growth as proposed by Pratontep et al. [28, 29], where the overlap area of the capture zones of neighboring islands is large.

Another important issue that we investigated is the role played by the kinetic energy of the impinging pentacene molecules in determining the island shape. To this end we 
considered the islands at low coverage where coalescence has not yet started (see Fig. $1(\mathrm{a}-\mathrm{d})$ ) and determined the fractal dimension for each growth condition. The island frac-
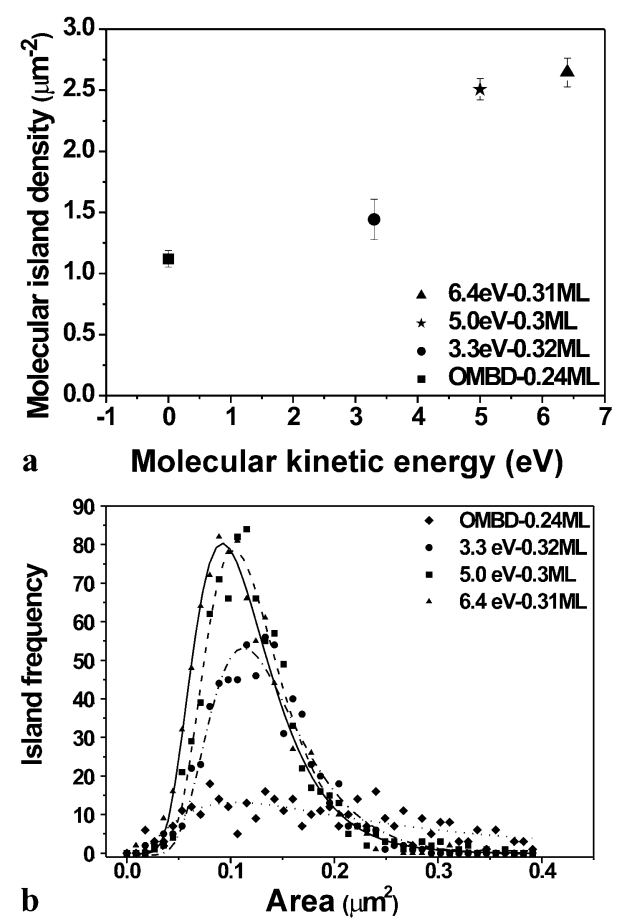

Fig. 2 a Molecular island density obtained for pentacene submonolayers grown on $\mathrm{SiO}_{x}$ by OMBD and by SuMBD at $E_{k}$ of 6.4, 5.0, and $3.3 \mathrm{eV}$; $\mathbf{b}$ molecular island size distribution for the same samples tal dimension $\left(D_{f}\right)$, as illustrated in Fig. 3(a-d), is calculated by the area-perimeter relationship $P=k A^{\frac{D_{f}}{2}}$, proposed in [30], where $P$ is the island perimeter, $A$ its area, $k$ a scaling constant and $D_{f}$ the fractal dimension. The mean $D_{f}$ turns out to be decreasing from $1.40 \pm 0.05$ for the SuMBD samples grown at $E_{k}=3.3 \mathrm{eV}$ to $1.39 \pm 0.04$ for those deposited with $E_{k}=5.0 \mathrm{eV}$ and $1.30 \pm 0.03$ for the layer formed when $E_{k}=6.4 \mathrm{eV}$. The data points become more scattered as $E_{k}$ decreases (see Fig. 3) due to the larger dispersion in island size distribution, but the $D_{f}$ values never approach those typical of a fractal island structure $\left(D_{f}=2\right)$, in agreement with the island morphology observable in Fig. 1. For the islands grown by OMBD, the mean $D_{f}$ cannot be correctly derived by one linear fit since the presence of two types of islands (see discussion Fig. 1(a) above) causes a much larger scattering of the data points. However, as shown in Fig. 3(d), if two different linear fittings are carried out for the majority and the minority species islands, one obtains a large mean $D_{f}=1.69 \pm 0.05$ for the small dendritic islands (reversed filled triangles in the figure) and $D_{f}=1.60 \pm 0.05$ for the data corresponding to the larger islands (labeled by filled diamonds). Dendritic or fractal islands occur naturally during growth in systems where island edge diffusion is restricted.

Figure 4 shows TM-AFM micrographs of layers obtained with the same deposition conditions as in Fig. 1 but for longer deposition times. When the coverage increases up to $\sim 0.5-0.65 \mathrm{ML}$, island coalescence is observed for all samples (Fig. 4(a-d)). Line scans (not shown) reveal that
Fig. 3 Mean fractal dimension $\left(D_{f}\right)$ obtained from a sample prepared by SuMBD at $6.4 \mathrm{eV}$ with

$D_{f}=1.30 \pm 0.03 ; \mathbf{b}$ sample prepared by SuMBD at $5.0 \mathrm{eV}$ with $D_{f}=1.39 \pm 0.04$; c sample prepared by SuMBD at $3.3 \mathrm{eV}$ with $D_{f}=1.40 \pm 0.05$; d sample prepared by thermal evaporation with the $D_{f}=1.60 \pm 0.05$ and $D_{f}=1.69 \pm 0.05 . S$ is the measured area and $P$ the perimeter of the individual island
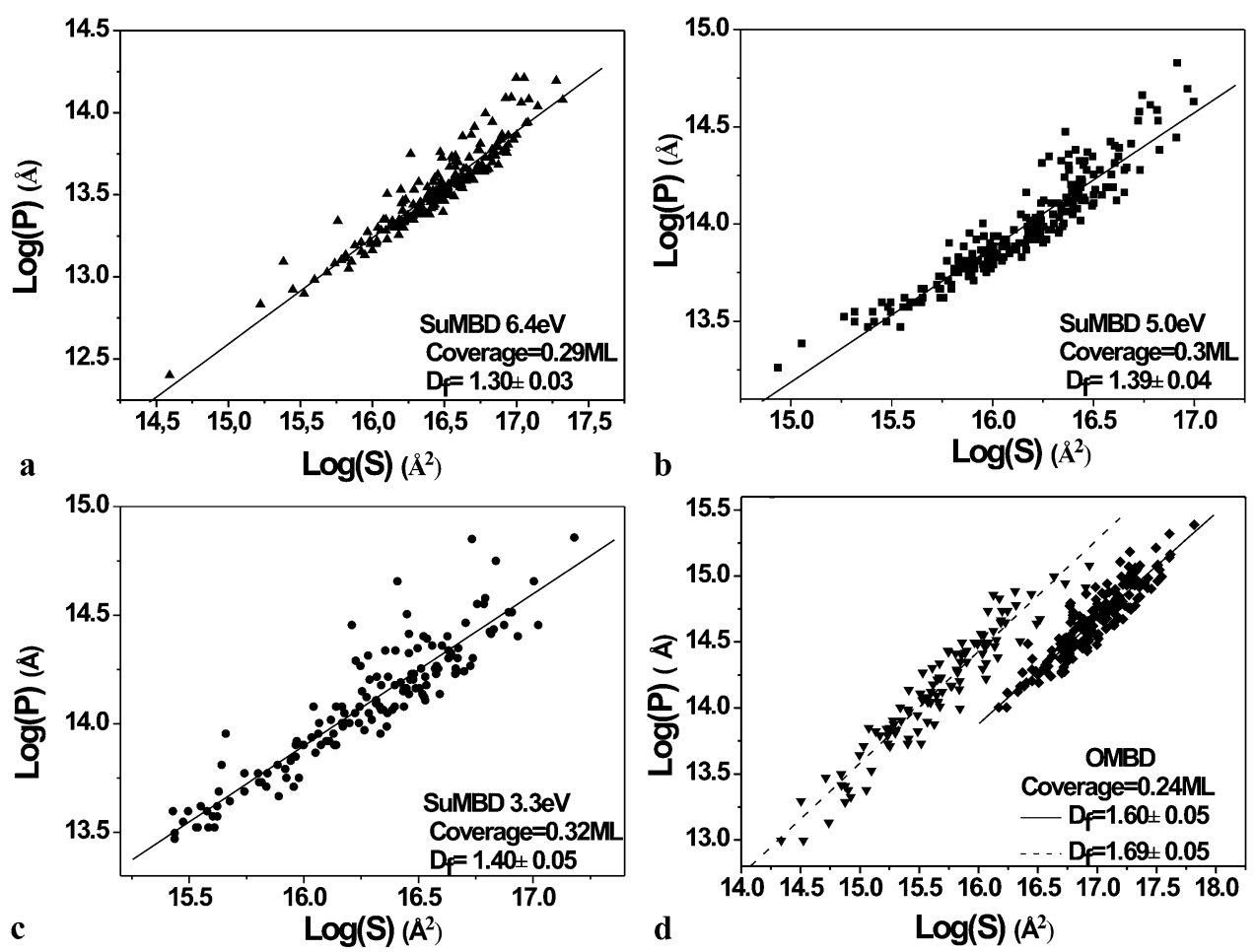
second-layer nucleation occurs for the OMBD grown pentacene at this coverage layer in agreement with a previous report [21]. In contrast, second-layer nuclei are extremely rare for all SuMBD samples. Close to the completion of the first monolayer (see Fig. 4(e-h)), all pentacene islands are connected to each other via coalescence, and grain boundaries appear. For all samples, the monolayer height is $1.5 \pm 0.2 \mathrm{~nm}$, confirming that pentacene molecules stand almost upright. Second- and third-layer nuclei, typical of
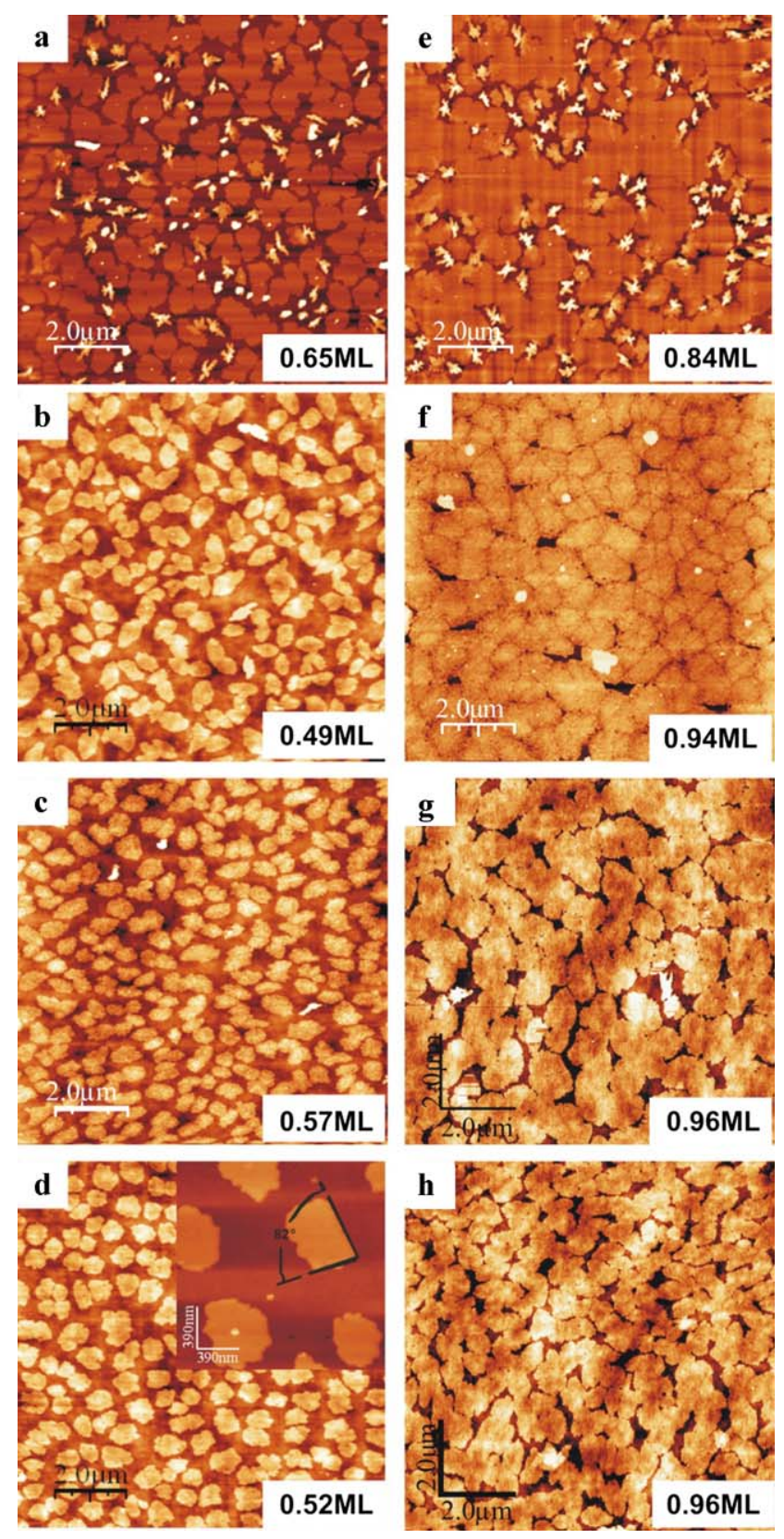

Fig. 4 Tapping mode AFM $\left(10 \times 10 \mu \mathrm{m}^{2}\right)$ micrographs of pentacene sub-monolayers grown by OMBD (a and e) and by SuMBD at $E_{k}=3.3 \mathrm{eV}$ (b and f), $E_{k}=5.0 \mathrm{eV}$ (c and $\mathbf{g}$ ), and $E_{k}=6.4 \mathrm{eV}$ (d and $\mathbf{h})$. The inset of (d) shows the angle of $82^{\circ}$ formed by two island edges
Vollmer-Weber growth mode, are now very clearly visible for the OMBD sample. While a few second layer islands are also present on top of the first layer of SuMBD samples grown at $E_{k}=3.3 \mathrm{eV}$ (Fig. 4(f)), SuMBD samples produced at higher $E_{k}$ hardly show any second-layer nuclei. This is a strong indication that at high $E_{k}$ the diffusivity of pentacene, after landing on the top of the first-layer islands, is sufficiently high to always sample the island edge, and its energy is large enough to overcome the edge barrier or "Schwoebel barrier" [31], which otherwise hinders the impinging pentacene from moving down to the substrate. Additionally, we often observe an island shape as shown in the inset of Fig. 4(d) with an angle of $\sim 82^{\circ}$ formed by two island edges, i.e., an angle very close to the one formed by the $a-b$ lattice vectors in the pentacene (001) crystal plane [32]. This suggests that the molecules can sample different adsorption sites before finally attaching to the island, and the island shape can become more anisotropic due the molecular diffusion along the island edges.

To learn more about the quality of the first monolayer and to determine the size of single-crystal domains we carried out TSM studies. Figure 5 compares contact mode AFM micrographs with the corresponding TSM images. The grain boundaries can be distinguished less well in the contact mode micrographs of Fig. 5 than in the tapping mode micrographs of Fig. 4, firstly because the surfaces of Fig. 4 is much flatter (no or much, much less second layer growth) and secondly because of the difference in operational mode and in spring constant of cantilever of the scanning tip $\left(\sim 0.58 \mathrm{~N} \mathrm{~m}^{-1}\right.$ for collecting the contact mode images and $\sim 40 \mathrm{~N} \mathrm{~m}^{-1}$ for the tapping mode ones).

The OMBD sample (Fig. 5(a, b)) shows a typical polycrystalline structure with random in-plane orientation of the single-crystalline domains in the first monolayer. The domain size reaches $1-2 \mu \mathrm{m}$ that corresponds closely to the average size of isolated pentacene islands before the onset of coalescence. The coalescence process seems not to influence the orientation alignment of merging islands (see arrows in Fig. 5(b)), in agreement with previous findings [27] so that a large number of grain boundaries is present in the first monolayer [33]. The second-layer islands, which cover about $1 / 5$ of the monolayer, show a typical dendritic shape with a mean fractal dimension $D_{f}=1.60 \pm 0.06,{ }^{1}$ and most of them are composed of several crystalline domains.

The SuMBD sample grown at $E_{k}=3.3 \mathrm{eV}$ (Fig. 5(c, d)) displays larger single-crystalline domains (see arrows in Fig. 5(d)) with more regular shape and sharp edges. The islands have merged after coalescence, and hence the number of grain boundaries is smaller than in the OMBD grown sample. Furthermore, in the second layer, covering about

\footnotetext{
${ }^{1}$ We determined this value by analyzing the shape of about 30 secondlayer islands analogously to what is described for first-layer islands.
} 


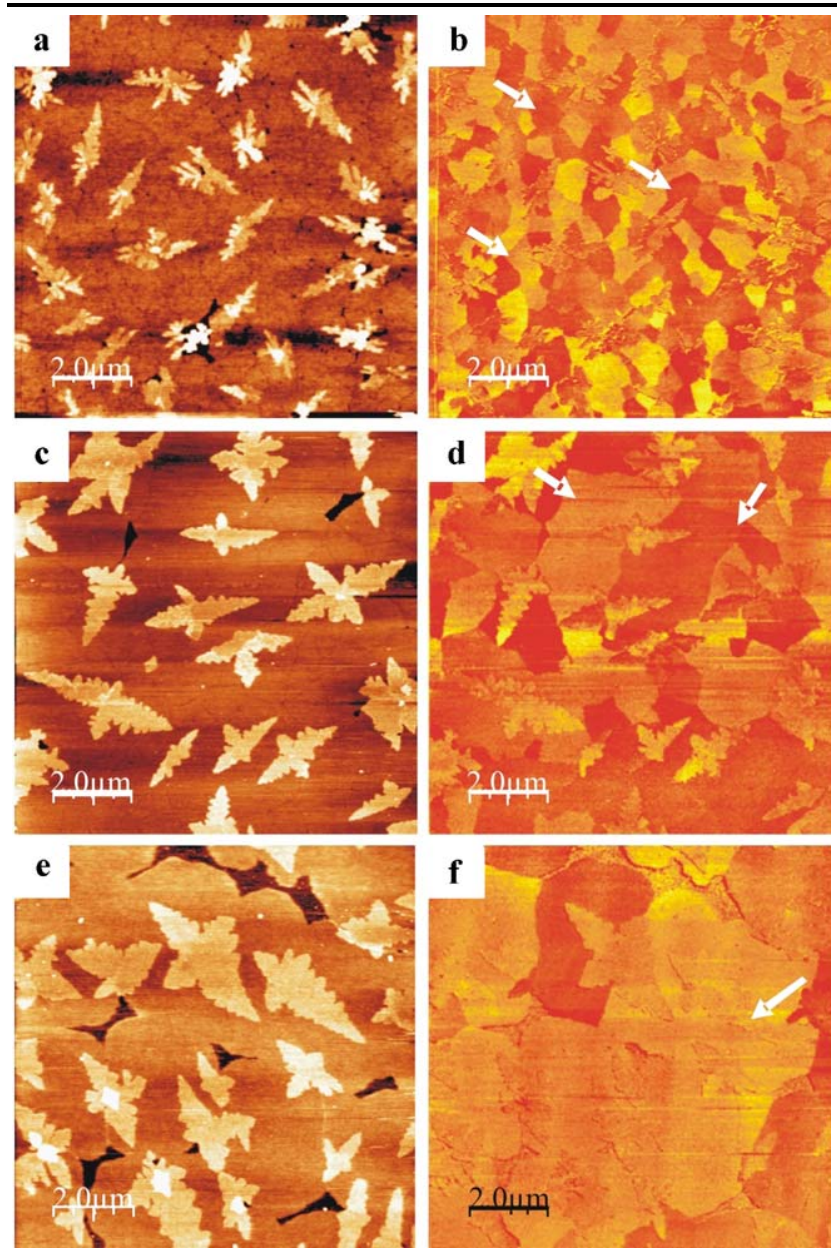

Fig. 5 Contact mode AFM height images (left panel) and transverse shear force micrographs (right panel) of pentacene monolayers grown on a silicon oxide surface by OMBD (a and $\mathbf{b}$, respectively) and by SuMBD where the molecules impinge on the surface with a kinetic energy of $3.3 \mathrm{eV}$ (c and d, respectively) and of $6.4 \mathrm{eV}$ (e and f, respectively)

$1 / 4$ of the first one, the mean fractal dimension of the islands is noticeably lower $\left(D_{f}=1.40 \pm 0.06\right.$, see footnote 1$)$ than in the OMBD sample, as observed also for the first monolayer (Fig. 3), and the percentage of single-crystalline islands much higher.

In the film grown at $E_{k}=6.4 \mathrm{eV}$ (Fig. 5(e, f)) the size of single-crystal domains in the first monolayer increases dramatically. The largest single-crystalline domain within the $10 \times 10 \mu \mathrm{m}^{2}$ scanning area, indicated by the arrow in Fig. 5 (f), spans $\sim 10 \mu \mathrm{m}$. The second layer covers about $1 / 3$ of the monolayer, and all of its molecular islands not only are larger and have a smaller $D_{f}=1.31 \pm 0.08$ (see footnote 1) than those of the other two samples, they also show a single-crystal structure. It is interesting to note that the shape of the island in terms of mean fractal dimension seems to be influenced by the kinetic energy much more strongly than by the nature of the substrate (silicon oxide for the first monolayer and pentacene for the second monolayer). There is a strong indication that, for the highest $E_{k}$, the majority of the second-layer islands grows epitaxially on the underlying crystal domain since they show the same friction force contrast. This implies that the island growth is highly correlated at high $E_{k}$, with a mechanism that favors orientation alignment of the islands before and during coalescence. On the basis of our experimental evidence and in particular in order to explain the generation of the observed large domains, we speculate that the reorientation of entire islands ${ }^{2}$ is part of the energy dissipation mechanism characteristic of SuMBD growth. Theoretical studies would be helpful to further clarify this question.

\section{Conclusions}

We have demonstrated that the kinetic energy of pentacene molecules, landing on a silicon oxide surface, plays a crucial role in determining island structure, shape, size distribution, and even the quality of the first monolayer and of upper layers. With increasing $E_{k}$, in the low-coverage regime, the islands revert from a fractal to a nonfractal structure, and the island shape becomes more anisotropic due the molecular diffusion along the island edges, while the much narrower island size distribution demonstrates a correlated island growth mode. The crystalline quality of the first monolayer is greatly improved when $E_{k} \geq 5.0 \mathrm{eV}$ presenting very few grain boundaries. To explain the formation of singlecrystalline domains spanning over $10 \mu \mathrm{m}$ in the monolayer, we propose a mechanism of reorientation of entire islands during coalescence as part of the energy dissipation induced by the high kinetic energy available at the impact.

Acknowledgements We gratefully acknowledge the precious technical support of A. Heeres, L. Venema, C. Corradi, M. Mazzola, M. Pola, and Dr. N. Tombros. We thank Dr. M. Lubomska, Dr. I. Arfaoui, Dr. F. Evangelista, Dr. R. Verucchi, and Dr. N. Coppedè for stimulating discussions, Dr. O.D. Jurchescu from the Solid State Chemistry group for purifying the pentacene powder, the Physics of Nanodevices group of the Zernike Institute for Advanced Materials in University of Groningen for use of the atomic force microscope. This work received financial support from the Dutch Foundation for Fundamental Research on Matter (FOM), from the Breedtestrategie program of the University of Groningen, from the Provincia Autonoma di Trento Project FOTOMINA, the Fondazione CARITRO Project CELTIC, and from the Italian Ministry of Education, University and Research (MIUR)—FIRB program project SYNERGY (RBNE03S7XZ). N.K. and J.Z. respectively acknowledge financial support by the Emmy Noether-Program (DFG) and by the Alexander von Humboldt Foundation for an individual research fellowship.

Open Access This article is distributed under the terms of the Creative Commons Attribution Noncommercial License which permits any noncommercial use, distribution, and reproduction in any medium, provided the original author(s) and source are credited.

\footnotetext{
${ }^{2}$ Note that major reorientation has been observed in other conditions, see, for example [34].
} 


\section{References}

1. S. Lee, B. Koo, J. Shin, E. Lee, H. Park, H. Kim, Appl. Phys. Lett. 88, 162109 (2006)

2. C.D. Dimitrakopoulos, P.R.L. Malenfant, Adv. Mater. 14, 99 (2002)

3. C. Reese, Z. Bao, Mater. Today 10, 20 (2007)

4. A. Di Carlo, F. Piacenza, A. Bolognesi, B. Stadlober, H. Maresch, Appl. Phys. Lett. 86, 263501 (2005)

5. R.A. Street, D. Knipp, A.R. Volkel, Appl. Phys. Lett. 80, 1658 (2002)

6. B. Nickel, R. Barabash, R. Ruiz, N. Koch, A. Kahn, L.C. Feldman, R.F. Haglund, G. Scoles, Phys. Rev. B 70, 125401 (2004)

7. A.C. Mayer, R. Ruiz, R.L. Headrick, A. Kazimirov, G.G. Malliaras, Org. Electron. 5, 257 (2004)

8. A.C. Mayer, A. Kazimirov, G.G. Malliaras, Phys. Rev. Lett. 97, 105503 (2006)

9. S. Schiefer, M. Huth, A. Dobrinevski, B. Nickel, J. Am. Chem. Soc. 129, 10316 (2007)

10. R. Ruiz, A. Papadimitratos, A.C. Mayer, G.G. Malliaras, Adv. Mater. 17, 1795 (2005)

11. L. Casalis, M.F. Danisman, B. Nickel, G. Bracco, T. Toccoli, S. Iannotta, G. Scoles, Phys. Rev. Lett. 90, 206101 (2003)

12. T. Toccoli, A. Palladoro, N. Coppedè, S. Iannotta, F. De Angelis, L. Mariucci, G. Fortunato, Appl. Phys. Lett. 88, 132106 (2006)

13. S. Pratontep, F. Nuesch, L. Zuppiroli, M. Brinkmann, Phys. Rev. B 72, 085211 (2005)

14. M. Kitamura, Y. Arakawa, J. Phys. Condens. Matter 20, 124011 (2008)

15. F.-J. Meyer zu Heringdorf, M.C. Reuter, R.M. Tromp, Nature 412, 517 (2002)

16. B. Stadlober, U. Haas, H. Maresch, A. Haase, Phys. Rev. B 74, 165302 (2006)

17. R. Ruiz, B. Nickel, N. Koch, L.C. Feldman, R.F. Haglund, A. Kahn, G. Scoles, Phys. Rev. B 67, 125406 (2003)
18. Y. Wu, T. Toccoli, N. Koch, E. Iacob, A. Pallaoro, P. Rudolf, S. Iannotta, Phys. Rev. Lett. 98, 076601 (2007)

19. A.W. Neumann, R.J. Good, in Surface and Colloid Science, vol. 11, ed. by R.J. Good, R.R. Stromberg (Plenum, New York, 1979)

20. O.D. Jurchescu, J. Baas, T.M. Palstra, Appl. Phys. Lett. 84, 3061 (2004)

21. R. Ruiz, B. Nickel, N. Koch, L.C. Feldman, R.F. Haglund, A. Kahn, G. Scoles, Phys. Rev. B 67, 125406 (2003)

22. S. Iannotta, T. Toccoli, J. Polym. Sci. B 41, 2501 (2003)

23. P. Milani, S. Iannotta, Cluster Beam Synthesis of Nano-Structured Materials (Springer, Berlin, 1999)

24. I. Horcas, R. Fernandez, J.M. Gomez-Rodriguez, J. Colchero, J. Gomez-Herrero, A.M. Baro, Rev. Sci. Instrum. 78, 013705 (2007)

25. R.M. Overney, H. Takano, M. Fujihira, W. Paulus, H. Ringsdorf, Phys. Rev. Lett. 72, 3546 (1994)

26. J.A. Last, M.D. Ward, Adv. Mater. 8, 730 (1996)

27. K. Puntambekar, J. Dong, G. Haugstad, C.D. Frisble, Adv. Funct. Mater. 16, 879 (2006)

28. S. Pratontep, M. Brinkmann, F. Nuesch, L. Zuppiroli, Phys. Rev. B 69, 165201 (2004)

29. M. Brinkman, S. Pratontep, C. Contal, Surf. Sci. 600, 4712 (2006)

30. P.A. Burrough, Principles of Geographical Systems for Land Resources Assessment (Clarendon, Oxford, 1986)

31. R.L. Schwoebel, J. Appl. Phys. 40, 614 (1969)

32. J.E. Northrup, M.L. Tiago, S.G. Louie, Phys. Rev. B 66, 121404 (2002)

33. J. Zhang, J.P. Rabe, N. Koch, Adv. Mater. 9999, 1-4 (2008)

34. M. Himmelhaus, M. Buck, M. Grunze, Mercury induced reorientation of alkanethiolates adsorbed on gold. Appl. Phys. B 68, 595 (1999) 